Frauke Reitemeier

\title{
Shakespeares Sommernachtstraum und die Boydell Gallery
}

Was zeichnet den Sommernachtstraum aus, daß es sich lohnt, im Zusammenhang mit dem ambitioniertesten englischen Publikationsvorhaben des 18. Jahrhunderts darüber zu arbeiten? Die Boydell Gallery wurde schließlich mit dem expliziten Ziel gegründet, die englische Historienmalerei anzuregen und zu neuer Größe und Bekanntheit zu verhelfen (Bruntjen 70 f.; Hammerschmidt-Hummel 1996). Angesichts dessen wäre es doch sicher lohnender, beispielsweise Macbeth zu untersuchen, denn schließlich handelt es sich dabei um ein Drama mit historischem Hintergrund; auch die Historien Shakespeares wären von Interesse, da sie naturgemäß die angemessensten Sujets für eine Umsetzung in der Boydell Gallery stellen. Der Sommernachtstraum hingegen ist ein halbes Märchen, eine der frühen leichten Komödien Shakespeares. Die Boydell-Illustrationen dazu können insbesondere im Vergleich zu denen anderer Shakespeare-Stücke doch kaum von Bedeutung sein.

Diese Einwände sind durchaus berechtigt. Die Beschäftigung gerade mit dem Sommernachtstraum hat jedoch auch Vorteile: Fast jeder kennt das Drama, und es ist sicher nicht übertrieben zu behaupten, daß die Vorstellungen, die jeder sich beim Lesen macht, sehr unterschiedlich sind. Tatsächlich bietet das Stück in der Umsetzung auf der Bühne eine enorme Bandbreite an Möglichkeiten - vermutlich mehr als die meisten anderen Shakespeare-Dramen, wie die folgenden beiden Beispiele 
zeigen sollen. Zwischen 2003 und 2006 wurde in den Herrenhäuser Gärten Shakespeares Sommernachtstraum in einer neuen Textversion von Heiner Lürig aufgeführt (Lürig/Kunze 2003). Die Kulisse im Herrenhäuser Gartentheater ist den Waldszenen des Dramas absolut angemessen; die realistische Umsetzung der Athener Akropolis ist hingegen etwas schwieriger zu bewerkstelligen, wenn Realismus überhaupt gewünscht wird bei einem Drama, das von Feen, Kobolden und Magie lebt. Oberon wird als Zwitterwesen dargestellt, als kriegerischer Mensch mit Widdergehörn; der Puck wirkt androgyn und hat zumindest äußerlich klare Züge eines Teufels (vgl. Abb. 3).

150 Jahre früher, 1856, inszenierte Charles Kean Shakespeares Sommernachtstraum im Drury Lane Theatre vor ausverkauften Haus. Die Bühnenbilder, die er verwendete, sind an Detailreichtum kaum zu überbieten (Kennedy 340-341). Die Athener Akropolis wurde gemäß der neuesten Ausgrabungsergebnisse gemalt; der Wald vor Athen zeigt im Hintergrund eine überaus realistische Landschaft, und sogar die Pflanzen im Vordergrund sind überzeugend. Kombiniert wurden diese Bühnenbilder mit einer ebenso opulenten Kostümausstattung.

Die Unterschiede zwischen den Inszenierungen sind sehr groß, und dennoch erheben beide den Anspruch, textgetreu oder zumindest dem Geist des Stücks gemäß zu sein. Solche Unterschiede hat sonst kaum ein Shakespeare-Stück aufzuweisen, und die ebenfalls höchst unterschiedlichen Interpretationsansätze zum Sommernachtstraum wurden hier noch nicht einmal in Ansätzen berücksichtigt. Allein das rechtfertigt eine Auseinandersetzung. Hinzu kommt, daß der Sommernachtstraum in der Boydell Gallery der ursprünglichen Konzeption gemäß nur mit einem Bild vertreten sein sollte (Proposal, 2; vgl. auch Bruntjen 254). Tatsächlich entstanden fünf verschiedene Bilder von drei verschiedenen Künstlern; vier der Gemälde liegen in Kupferstichen vor. Offenbar war der Sommernachtstraum auch für die an der Gallery beteiligten Künstler hochinteressant.

Das Ziel dieser Abhandlung ist es zu zeigen, welche Stellung den BoydellIllustrationen im Kontext der Rezeptionsgeschichte des Sommernachtstraums im England des 18. Jahrhunderts zukommt. Dazu ist zunächst ein Blick auf die TextRezeption wichtig, der nachzeichnet, welche Vorstellungen sich Leser und Regisseure bis ins späte 18. Jahrhundert vom Sommernachtstraum machen; außerdem werden die vor den Boydell-Illustrationen entstandenen Bilddarstellungen zum Sommernachtstraum analysiert, um daran abschließend Traditionen und Neuansätze in den Bildern der Boydell Gallery festzumachen. 


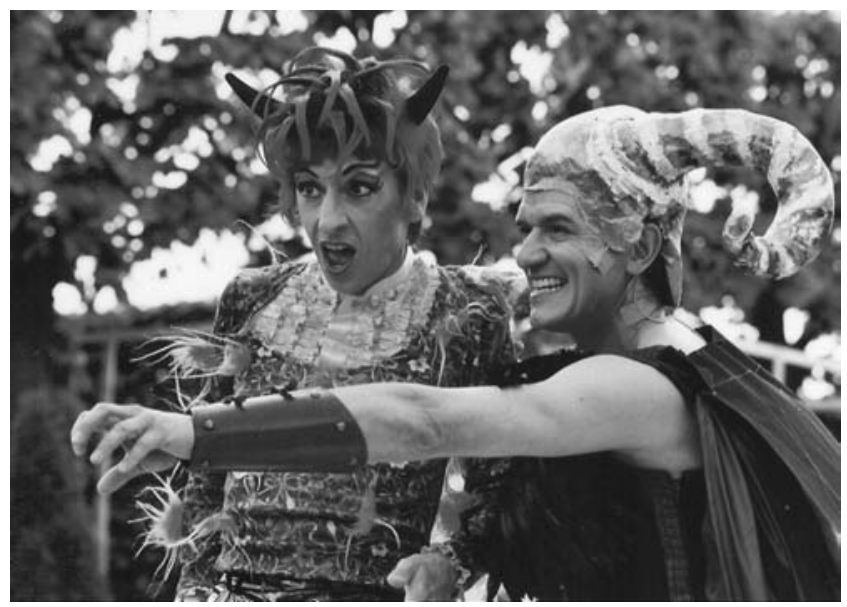

Abb. 3: Puck und Oberon (Szenenfoto der Aufführung von Ein Sommernachtstraum. Das Musical in den Herrenhäuser Gärten 2002)

\section{Zur Textrezeption}

Die Handlung des Sommernachtstraums spielt sich auf mehreren Ebenen ab (Pfister 406-409). Eine Handlungsebene betrifft Theseus und Hippolyta sowie die vier verliebten Athener; hier ist die ,richtige ${ }^{6}$ oder ,falsche' Liebe und die Wahl des richtigen Lebenspartners der Mittelpunkt. Theseus und Hippolyta haben sich bereits gefunden, die vier jungen Athener noch nicht endgültig.

Der zweite Handlungsstrang betrifft die vier Handwerker, die das Theaterstück für die Hochzeitsfeierlichkeiten von Theseus und Hippolyta einstudieren. Zunächst scheint hier thematisch keine Verbindung zwischen den beiden Handlungsebenen vorzuliegen. Aber auch hier geht es um Liebe, zumindest in dem Theaterstück: Pyramus und Thisbe sind zwei Liebende, die voneinander getrennt sind, das aber nicht hinnehmen wollen und sich heimlich im Wald treffen. Damit stellt das Stück eine Reflexion auf Hermia und Lysander dar; auch in ihrem Fall greift ein im Wald lebendes Wesen in die Liebesbeziehung ein: Pyramus und Thisbe werden von einem Löwen gestört - dem König der Tiere - , der für das tragische Ende verantwortlich ist. In die Beziehung zwischen Hermia und Lysander greift der König der Elfen ein, Oberon, und sorgt für ein gutes Ende.

Damit ist bereits der dritte Handlungsstrang angesprochen, der um die Feen oder Elfen. Auch hier steht Liebe im Mittelpunkt: Zwei, die zueinander gehören, Oberon und Titania, haben sich über einen relativ nichtigen Anlaß zerstritten, und keiner ist bereit, dem anderen nachzugeben. Oberons Entscheidung in seiner Welt wie in der der Menschen einzugreifen verschiebt das Gleichgewicht zwischen den 
beteiligten Lebewesen - Menschen wie Elfen - und sorgt letztlich für eine neue Balance.

Drei Bereiche sind also in der ursprünglichen Fassung des Dramas von Bedeutung: Zum einen das Thema - die richtige/falsche Liebe -, zum anderen die Handwerker, die einerseits Objekt des Eingreifens der Elfen sind, andererseits das rein komische Element im Drama darstellen, und schließlich die Elfen und ihre Einmischung in die Menschenwelt.

Welche Reaktionen Shakespeares Zeitgenossen auf dieses Stück zeigten, ist nicht überliefert. Es ist nicht einmal ganz klar, wann der Sommernachtstraum verfaßt und zuerst aufgeführt wurde (Pfister 404). Sicher scheint nur, daß die letzte Aufführung zu Lebzeiten Shakespeares 1604 stattfand. Danach scheint das Stück lange Zeit in Vergessenheit geraten zu sein; teils hängt das damit zusammen, daß sich mit der Thronbesteigung der Stuarts durch James I. 1603 der Theatergeschmack des Publikums etwas änderte: Rachetragödien werden populärer. Ein weiterer Grund liegt darin, daß die Puritaner in den 1620er Jahren zunehmend an Einfluß gewinnen, für die weltliches Theater inakzeptabel ist. 1642 werden die Theater geschlossen, und für die nächsten zwanzig Jahre sind Theateraufführungen nur in privaten Räumlichkeiten und nur vor kleinem Publikum möglich. Angesichts der politischen Situation wird der Sommernachtstraum kaum dazu gehört haben, aber über das Theaterleben der Zeit ist nur sehr wenig bekannt.

1660 wird mit Charles II. die Monarchie wieder in England eingeführt; Charles II. hatte fast sein ganzes Leben am französischen Hof verbracht. Mit ihm kehren englische Adlige nach England zurück, die wie er die französische Kultur und Lebensweise kennen- und schätzengelernt haben. Anders als in den Regierungszeiten von Elizabeth I. und James I. wird das Theater nun vorwiegend von den Adligen des Hofs protegiert, und deren Theatergeschmack ist an der französischen Dramenproduktion ausgerichtet. Bis sich die einheimischen englischen Autoren dem angepaßt haben, vergehen einige Jahre; in dieser Zeit werden auch ältere Stücke aufgeführt, die auf den neuen Geschmack abgestimmt inszeniert werden.

Im Fall des Sommernachtstraums ist nicht ganz klar, welche Form diese Ausrichtung annahm. Samuel Pepys, der 1662 eine Aufführung des Sommernachtstraums der Theatertruppe unter Thomas Killigrew besuchte, ist zumindest nicht besonders beeindruckt - aber Pepys ist ohnehin kein besonderer Liebhaber des Theaters. Etwas mißgestimmt kommentiert er, daß allenfalls Tanz und Musik akzeptabel gewesen wären (Pepys 208). Ganz offensichtlich beschloß Killigrew, das Stück durch zusätzliche Musik- und Tanzeinlagen zu verbessern; welchen Umfang das hatte, bleibt allerdings offen. Eine der Inszenierung zugrunde liegende Textversion ist nicht überliefert. Aber der verstärkte Einbezug von Musik und Tanz zeigt sich auch in der folgenden, überaus erfolgreichen Verarbeitung des Sommernachtstraums von 1692 durch Thomas Betterton und seine Theatergruppe: Die Musik zum Stück wird von Henry Purcell beigesteuert, und die Neuinszenierung wird 
unter dem Titel The Fairy Queen (Die Elfenkönigin) angekündigt. Allerdings ist im Vergleich mit Shakespeares Text die Handlung verändert worden: Liebe ist durchaus noch ein Thema der Fairy Queen, aber unter gänzlich neuen Vorzeichen. Viel wichtiger als die geänderte Themensetzung ist jedoch die äußere Umarbeitung: The Fairy Queen ist eine Semioper; der Schwerpunkt liegt auf den Möglichkeiten, die die Opernbühne im Gegensatz zur Theaterbühne in der Darstellung von Exotik und Opulenz hat. Besonders deutlich wird dies im letzten Akt: Die Szene zeigt einen chinesischen Garten - komplett mit Früchten, Pflanzen und Tieren, darunter Affen, die später einen eigenen Tanz aufführen (Muller and Muller 676 f.) - , in der ein chinesisches Paar die Freuden der Liebe in Freiheit besingt. Die Elfen sind hier Teil der Exotik; ein innerer Zusammenhang der einzelnen Handlungsteile ist weitgehend unwichtig.

Vierundzwanzig Jahre später, 1716, greift Richard Leveridge den ursprünglichen Stoff wieder auf: Auf der Basis des Sommernachtstraums kritisiert er das zeitgenössische Musiktheater. Dreißig Jahre später, 1745, wird sein Stück in umgearbeiteter Fassung unter dem Titel Pyramus and Thisbe: A Mock-Opera (Pyramus und Thisbe: Eine Scherzoper) neu inszeniert (Stone 467). Dabei wird die bereits in der früheren Version angelegte Kritik an der zeitgenössischen Oper verschärft. Die Personalliste ist deutlich kürzer und umfaßt lediglich die Handwerker. Die eigentlich viel wichtigeren Figuren verbergen sich hinter „the other Parts“ ([Leveridge] viii): der master, der Theaterinhaber, sein Souffleur (prompter), der hier auch die Rolle eines Bühnenmanagers spielt, und das geladene Publikum. Letzteres besteht aus Mr. Semibrief und zwei gentlemen, von denen einer gar nichts von der heimischen englischen Art der Unterhaltung hält, denn die englische Sprache ist nicht für die Oper geeignet und in England gibt es ohnehin keine passenden, gut ausgebildeten Opernsänger. Trotz der Bemühungen Semibriefs sind seine beiden Gäste von der Vorstellung nur wenig begeistert ([Leveridge] 11, 13). Die HandwerkerOper, die ihnen vorgeführt wird, bestätigen diese Vorurteile offensichtlich auch.

Zwischen der Restauration des Königshauses 1660 und 1745 vollzieht sich also eine drastische Veränderung mit dem Sommernachtstraum: Die HandwerkerHandlung wird herausgelöst und durchgehend als einziges originales Handlungselement beibehalten; dabei entfallen jedoch die adligen Figuren und ihre Kommentare völlig, ebenso wie die Elfen zunächst nur der Exotik halber beibehalten bleiben und später gänzlich gestrichen werden. Statt der ursprünglichen Schwerpunktsetzung auf die Frage nach der angemessenen Liebe rückt insbesondere im 18. Jahrhundert die Kritik am zeitgenössischen Musiktheater als Thema in den Vordergrund. Wie kommt das? Wer oder was ist verantwortlich dafür, daß der Sommernachtstraum in seiner eigentlichen Form knapp 100 Jahre lang nicht mehr inszeniert wird? Die Gründe sind in der Literatur- und Theatertheorie zu suchen.

Shakespeares Dramen stellen für die Literaturtheoretiker wie für die Literaturkritik des 18. Jahrhunderts ein ernstzunehmendes Problem dar. Der französische Literaturgeschmack, der im Zug der Restauration des Königshauses nach England 
importiert wurde, ist an Aristoteles' Regelpoetik ausgerichtet, und Shakespeares Dramen entziehen sich dieser Poetik weitgehend. In Charles Gildons Vorwort zu einer der ersten ernstzunehmenden Shakespeare-Ausgaben des 18. Jahrhundert beschreibt er ausführlich das Muster, dem Dramen zu folgen haben (Gildon 1710a, xxix-lix). Die Hauptpunkte, die er für die Tragödie anspricht, betreffen die drei Einheiten der Zeit, des Handlungsortes und der Handlung selbst, die Darstellungsart des Dramas - Mimesis, Darstellung der Realität - und den kathartischen Zweck, die ,Reinigung' des Zuschauers durch Identifikation mit den Figuren. Gildon verwendet allerdings dabei so viel Raum auf die Tragödie, daß für die Komödie keine Zeit mehr bleibt; er konstatiert lediglich, daß beide Gattungen viele der Regeln miteinander teilen (Gildon 1710a, lix). In seiner die Ausgabe abschließenden, sehr knappen literaturkritischen Analyse der einzelnen Dramen findet Gildon eine ganze Reihe von Fehlern im Sommernachtstraum: Um das Problem der nicht realistischen Elfen und der Verwendung von Magie etwa versucht er durch den Verweis auf die Bibel herumzukommen, die ja auch von Geistern berichtet (Gildon 1710b, 265); die Einheit der Zeit von einem einzigen Tag wird nicht eingehalten, und auch die Handlung, die der neoklassischen Auffassung nach eine Einheit darstellen soll, ist nicht regelgemäß (Gildon 1710b, 315). Hinzu kommt, daß Theseus als Held aus der griechischen Mythologie nicht zum Personal einer Komödie gehören sollte. Den Regeln gemäß kann Gildon den Sommernachtstraum nicht als gutes Drama betrachten; dennoch stellen die lyrischen Passagen und die im Drama verarbeitete klassisch-antike Bildung Gründe dar, Shakespeare zu entschuldigen (Gildon 1710b, 316-320).

Einige der Regelverstöße könnten schon durch textuelle Anpassungen zumindest abgemildert werden. Anders sieht es mit den magischen, übernatürlichen Elementen aus: Sie sind ein integraler Bestandteil des Sommernachtstraums und lassen sich nicht einfach austauschen.

Nun finden Elfen und Feen durchaus Eingang in die Literatur der Zeit. Aber ihr „Einsatzgebiet“ ist weitgehend auf eine bestimmte Gattung beschränkt, auf das Märchen, das im 18. Jahrhundert als eine Textart betrachtet wird, mit denen Kinder unterhalten werden. Märchen, allegorische Erzählungen oder Feengeschichten wie beispielsweise diejenigen der Comtesse d'Aulnoy, sind keine ,ernste ${ }^{6}$ Literatur. Die Elfen im Sommernachtstraum sind daher nicht nur aus regelpoetischen, sondern auch aus gattungstheoretischen Gründen schwer in einer Inszenierung zu rechtfertigen, die ernstgenommen werden will.

Eine Änderung ergibt erst sich um die Mitte des 18. Jahrhunderts. 1755 inszeniert der berühmte Schauspieler und Regisseur David Garrick den Sommernachtstraum wiederum als Oper unter dem Titel The Fairies (Die Elfen). Anders als seine Vorgänger streicht er das komische Element, die Handwerkerhandlung, gänzlich; die Handlungsstränge um Theseus' und Hippolytas Hochzeit, um die vier Athener Liebenden sowie Oberon und Titania sind jedoch zu großen Teilen unangetastet. 
Lediglich der Text wird gestrafft, teils modernisiert und mit Liedern und Rezitativen ergänzt (Stone 469-472).

Garricks enorm erfolgreiche Inszenierung wird acht Jahre später, 1763, durch eine fünfaktige Opernfassung von Garricks Partner George Colman gefolgt, die die Handwerkerhandlung und mehr Textzeilen aus dem ursprünglichen Drama enthält, das Publikum jedoch nicht zu begeistern vermag (Stone 473). Nur in einer verkürzten Form, als Nachspiel zu anderen Inszenierungen, wird The Fairies in den nächsten vierzehn Jahren immer wieder aufgeführt (Stone 482). Dennoch handelt es sich bei Garricks wie bei Colmans Versionen stets um Opernversionen, nicht um Shakespeares Drama als Sprechtext: Immer noch steht nicht die Originalfassung im Mittelpunkt, weder im Hinblick auf die Handlungsstränge noch auf Gehalt oder Konzeption.

\section{Frühe Illustrationen zum Sommernachtstraum}

Im Lauf des 18. Jahrhunderts gewinnt Shakespeare allerdings zunehmend den Status eines einzigartigen weil ,originalen' Autors (Friedman 22-23). Gerade seine Unbekümmertheit im Umgang mit den aristotelischen Dramenregeln wird - oft in Verbindung mit seiner Sprache - als Zeichen einer Besonderheit gesehen, die die englische Literatur vor der französischen (langweiligen, weil nur regelgetreuen) Literatur auszeichnet. Greifbar wird dies vor allem in den zahlreichen Shakespeare-Gesamtausgaben, die im Lauf des 18. Jahrhunderts in England herausgegeben werden. Den Anfang macht Nicholas Rowe 1709 mit einer ersten kritischen Ausgabe; ,kritisch“ heißt in diesem Fall, daß die Dramentexte teils mit Anmerkungen und die Gesamtausgabe mit einem Vorwort versehen wird, das in diesem Fall der renommierte Autor und Übersetzer Alexander Pope beisteuert. Die erfolgreiche Ausgabe ist mit einem Frontispiz versehen, das Shakespeare zeigt, und jedes einzelne Stück ist von einer Illustration begleitet (vgl. zur Ausgabe allgemein Hammerschmidt-Hummel 2003, 1: 13-14). Die hochformatige Illustration zum Sommernachtstraum zeigt die Konfrontation zwischen Oberon und Titania in einer amönen Landschaft (Shakespeare 1709, Vorblatt; Abb. 4): Der Wald, in dem sich die Lichtung im Vordergrund befindet, ist ein lichter Mischwald, im Hintergrund schlängelt sich ein Fluß, in dem sich die Bäume spiegeln, und am oberen Bildrand ist der aufgehende Mond (oder die untergehende Sonne) zu sehen. Oberon wird in Haltung und Kleidung wie ein Krieger aus der klassischen Antike gezeigt; einer seiner Begleiter - vielleicht der Kobold? - hält seinen Umhang wie eine Schleppe. Titania ist genauso groß wie Oberon, und sie scheint wie Oberon in antiker Kleidung dargestellt zu sein; auch ihr ist eine Elfe zugeordnet, die ihren Umhang hält. $\mathrm{Da}$ die beiden sich nicht freundlich gesonnen sind, zeigt sich in ihrer Körperhaltung - Oberon steht leicht von ihr abgewandt, während Titania sich ihm deutlich stärker zuwendet - und vor allem in ihrer Gestik. 


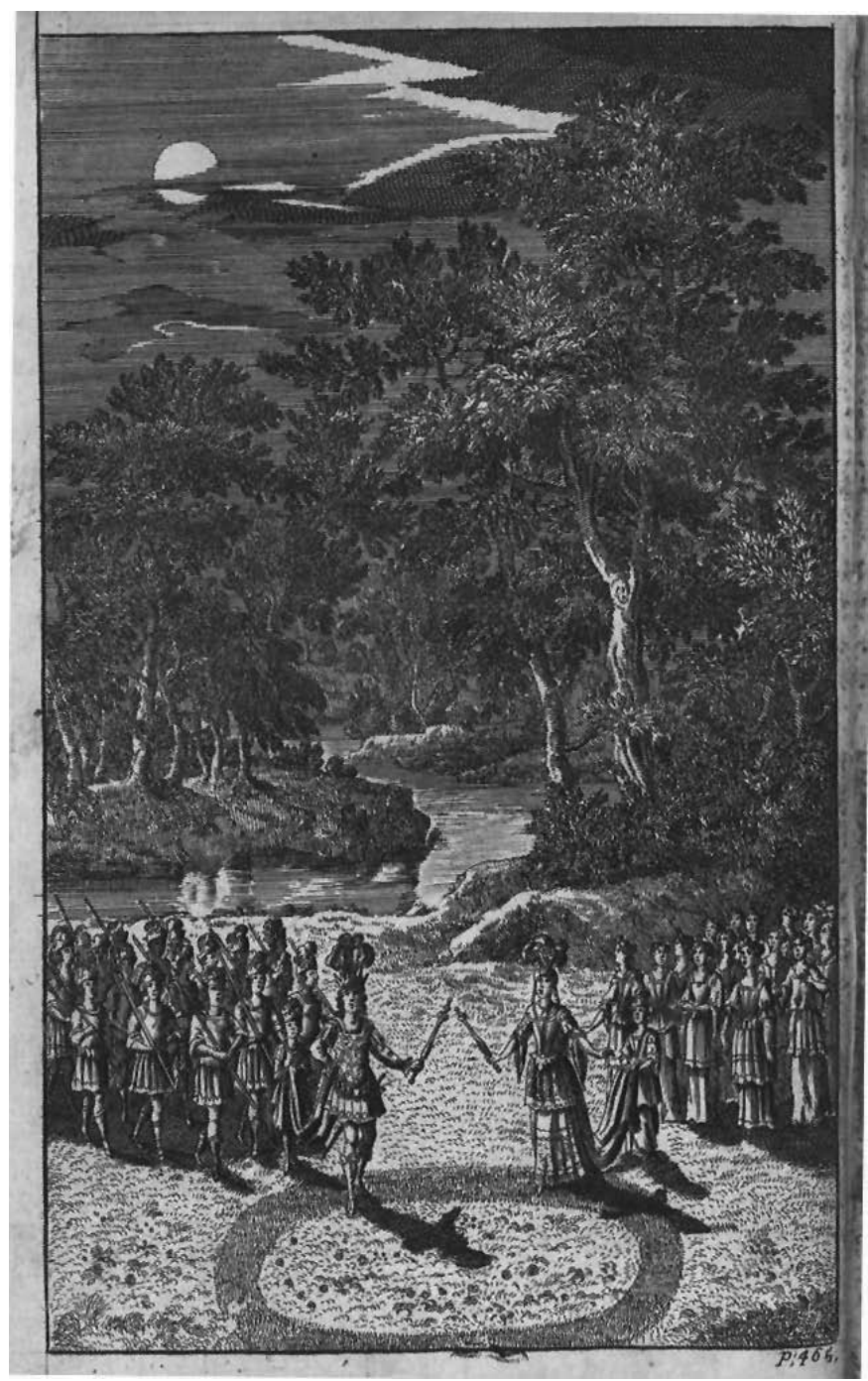

Abb. 4: Titania und Oberon (Frontispiz: Sommernachtstraum, London 1709) 


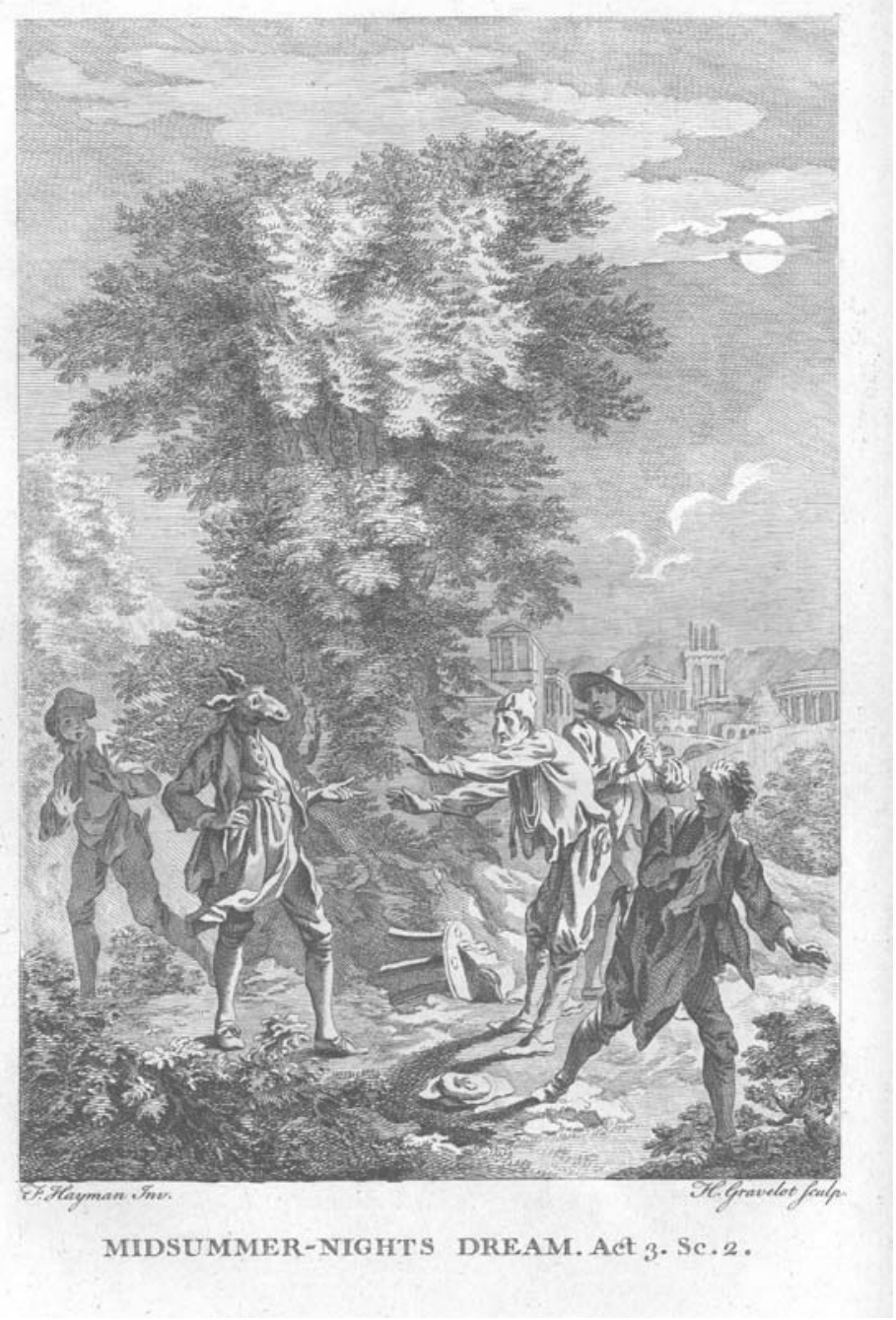

Abb. 5: F. Hayman / H. Gravelot, A Midsummer Night's Dream (Act III, Scene 2; Frontispiz: Sommernachtstraum, London 1770) 


\section{MIDSUMMIFIRS NIGHET DIRTEAMT.}

Act $\mathrm{TI}$.

Scenell.

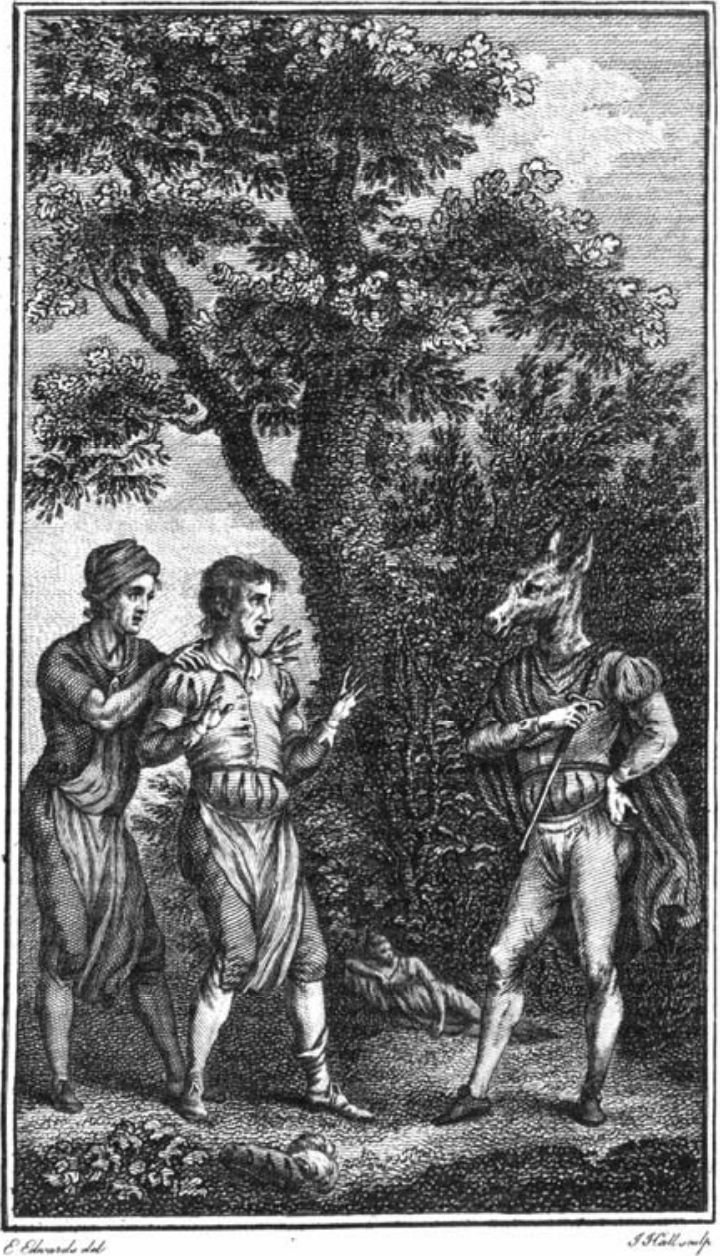

Abb. 6: Anon. [J. Hall?], "O Bottom, thou art chang'd" (Act III, Scene 2; Frontispiz: Sommernachtstraum, London 1773) 
Beide tragen einen Stab, vielleicht ein Szepter, das zumindest bei Oberon an ein gezogenes Schwert erinnert. Insgesamt ist die Darstellung von der zeitgenössischen Theaterkunst beeinflußt (Friedman 24); das zeigt sich insbesondere in der Haltung der beiden zentralen Figuren. Daß hier Elfen und nicht Menschen gezeigt werden, ist in erster Linie an dem Feenring im Vordergrund zu erkennen. ${ }^{91}$

Rowes Ausgabe ist sehr populär und darüber hinaus lange Zeit die einzige auf dem Buchmarkt erhältliche Gesamtausgabe. Erst 1744 unternimmt Sir Thomas Hanmer den Versuch, eine Ausgabe mit neuen Illustrationen herauszubringen. Viele der Gemälde, auf denen die in die Ausgabe eingearbeiteten Stiche beruhen, trägt der Maler Francis Hayman bei (Friedman 211). Haymans Gemälde zum Sommernachtstraum - ebenfalls ein Hochformat - stellt eine Szene im 3. Akt dar (Shakespeare 1770: 1, Frontispiz; Abb. 5): den Moment, die Handwerker den verzauberten Zettel sehen und vor ihm fliehen. Hier, anders als in der Illustration zu Rowes Ausgabe, ist der Ort der Handlung nicht auf einer abgeschiedenen Lichtung im Wald; die Stadt Athen ist rechts im Hintergrund deutlich zu erkennen. Gegenüber davon ist auf der anderen Seite der Wald angedeutet. Dominant im Mittelgrund des Bildes steht ein hochgewachsener Laubbaum, unter dem sich die Handwerker getroffen haben. Links vor dem Baum steht Zettel in Rednerhaltung; die linke Hand unterstreicht seine Worte, während er die rechte Hand in Unterstützung der dozierenden Haltung in die Hüfte gestemmt hat. Links und rechts von ihm sind seine Kollegen zu sehen, deren Haltung und Gesichtsausdruck soweit erkennbar - anschaulich ihr Entsetzen und ihre Abwehr zeigen; am deutlichsten wird das bei der Figur unmittelbar rechts von Zettel: Schlucker der Schneider, dem eine Schere aus der Hosentasche schaut, streckt beide Arme abwehrend gegen Zettel und schiebt gleichzeitig seinen Körper so weit wie möglich von ihm weg. Rechts davon faltet ein anderer um Schutz flehend die Hände. Welchen Aufruhr Zettels Verzauberung ausgelöst hat, ist zudem an dem umgestürzten Tisch im Hintergrund zu erkennen. Auch hier ist die Körperhaltung der einzelnen Figuren noch deutlich an die für Schauspieler üblichen Bewegungsmuster angelehnt, aber das ganze Bild zeigt die Dramatik, die das Eingreifen des Kobolds für die Handwerker hat. Die hohe Qualität läßt sich am besten erkennen, wenn dieser Stich mit einer thematisch ähnlichen Abbildung aus einer anderen ShakespeareAusgabe verglichen wird (Shakespeare 1773-1774: Frontispiz; Abb. 6) (vgl. zu Haymans Shakespeare-Illustrationen allgemein Hammerschmidt-Hummel 2003, 19): Hier sind die beiden Handwerker links zwar auch entsetzt, wie auch der rechts stehende Zettel in einer Rednerpose gezeigt wird, aber insgesamt wirkt das Bild viel statischer und wenig überzeugend.

91 Nach Abschluss der Überarbeitung dieses Textes wurde die Dissertation von Dorothea Gerkens unter dem Titel Elfenbilder - Traum, Rausch und das Unbewusste. Die Erkundung des menschlichen Geistes in der Malerei des 18. und 19. Jahrbunderts veröffentlicht (Berlin: Reimer, 2009), die den Darstellungen von Elfen in der englischen Malerei nachgeht. Die Dissertation konnte leider aus Zeitgründen nicht mehr eingearbeitet werden. 


\section{Der Sommernachtstraum in der Boydell Gallery}

Erst mit dem Projekt von John Boydell wird wieder ein Anlauf unternommen, eine illustrierte Prachtausgabe der Werke Shakespeares zu schaffen. Die Entwicklung der Bildillustrationen in den Shakespeare-Ausgaben ist jedoch überaus interessant, insbesondere im Vergleich mit der Schwerpunktsetzung der Sommernachtstraum-Inszenierungen: Während dort in der ersten Hälfte des 18. Jahrhunderts der Handlungsstrang um Oberon und Titania völlig vernachlässigt wird, ist es gerade eine solche Szene, die in den Shakespeare-Ausgaben zur Illustration verwendet wird: Offenbar war für den Herausgeber wie für den Verleger die Konfrontationsszene zwischen Oberon und Titania interessanter oder wichtiger als die Handwerker-Handlung. Diese wird erst für die 1744 erschienene Prachtausgabe Hanmers zur Bebilderung des Sommernachtstraums herangezogen, während zugleich dieser Handlungsstrang für die Inszenierungen des Dramas zentral ist. Die elf Jahre später einsetzende neue Schwerpunktsetzung unter der Federführung von David Garrick hingegen ist offenbar erst in den Bildern der Boydell Gallery greifbar. Hier stehen nur noch die Elfen bzw. der Kobold Robin Goodfellow, der Puck, im Mittelpunkt der Darstellung.

Das erste Gemälde zum Sommernachtstraum, das Boydell nach einigen Schwierigkeiten akquirieren konnte (Postle 256-258), ist Sir Joshua Reynolds Puck (Abb. 6). Es zeigt weitgehend nackten kleinen Jungen auf einem Pilz, der den Betrachter anlächelt. In seiner rechten Hand hält er einen Strauß Stiefmütterchen, die Pflanze love-in-idleness, die Oberon für seine Verzauberung Titanias und Demetrius' verwenden will. Mit der anderen Hand winkt er fröhlich. Unmittelbar hinter dem Puck wächst ein Baum in die Höhe, von einer Efeuranke umschlungen; am linken Bildrand sind weitere Bäume zu sehen, während rechts eine Gestalt mit Eselskopf und eine schlafende nackte Frau zu erkennen ist (Friedman 123). Neben seinem Gesichtsausdruck sind seine spitzen Ohren das Einzige, das ihn als Kobold auszeichnet. Im Mittelpunkt des Bildes steht deutlich das Kind, was im Stich noch klarer zu erkennen ist, da hier durch die notwendige Reduktion in der Farbgebung die Hell-Dunkel-Kontraste stärker herausgearbeitet werden müssen: Der Hintergrund ist in dunklen Grauschattierungen gehalten, lediglich die schlafende Titania sticht heraus, während der Puck im Vordergrund hell und wie von Licht beschienen wirkt. 


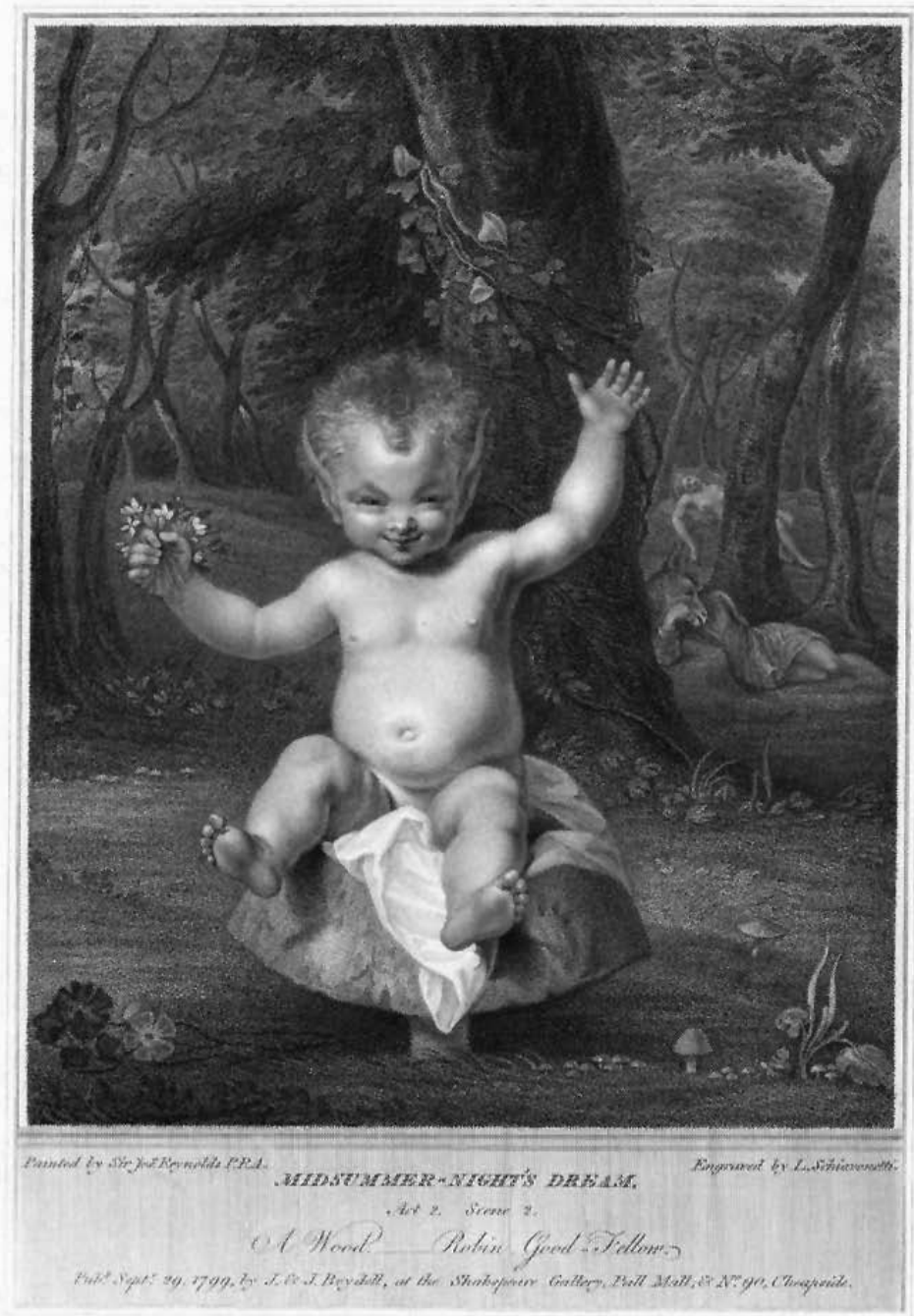

Abb. 7: J. Reynolds / L. Schiavonetti, A Midsummer Night's Dream (Act II, Scene 2): A Wood. Robin Good-Fellow 


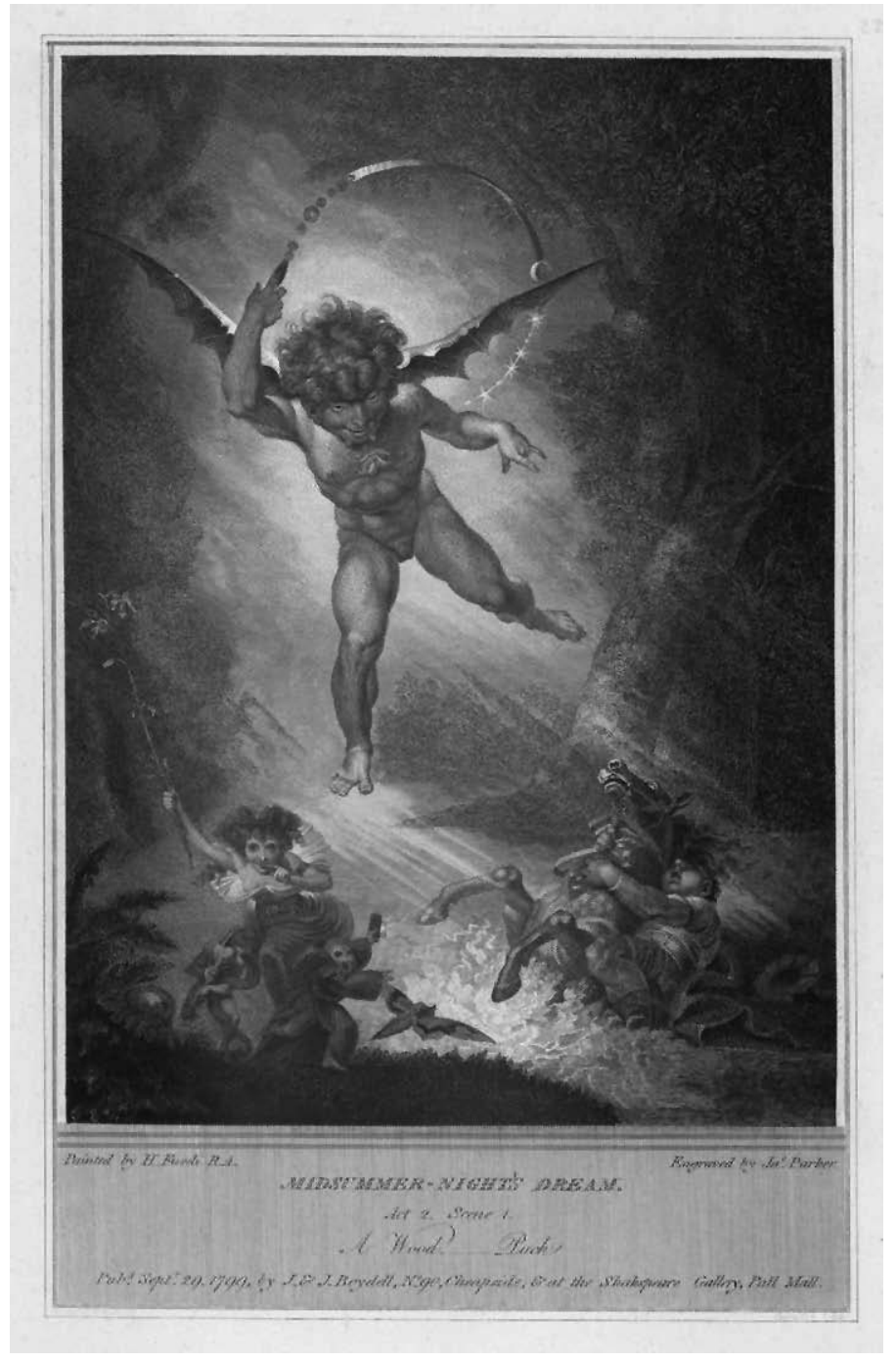

Abb. 8: J. H. Füssli / J. Parker, A Midsummer Night's Dream (Act II, Scene 1): A Wood. Puck. 


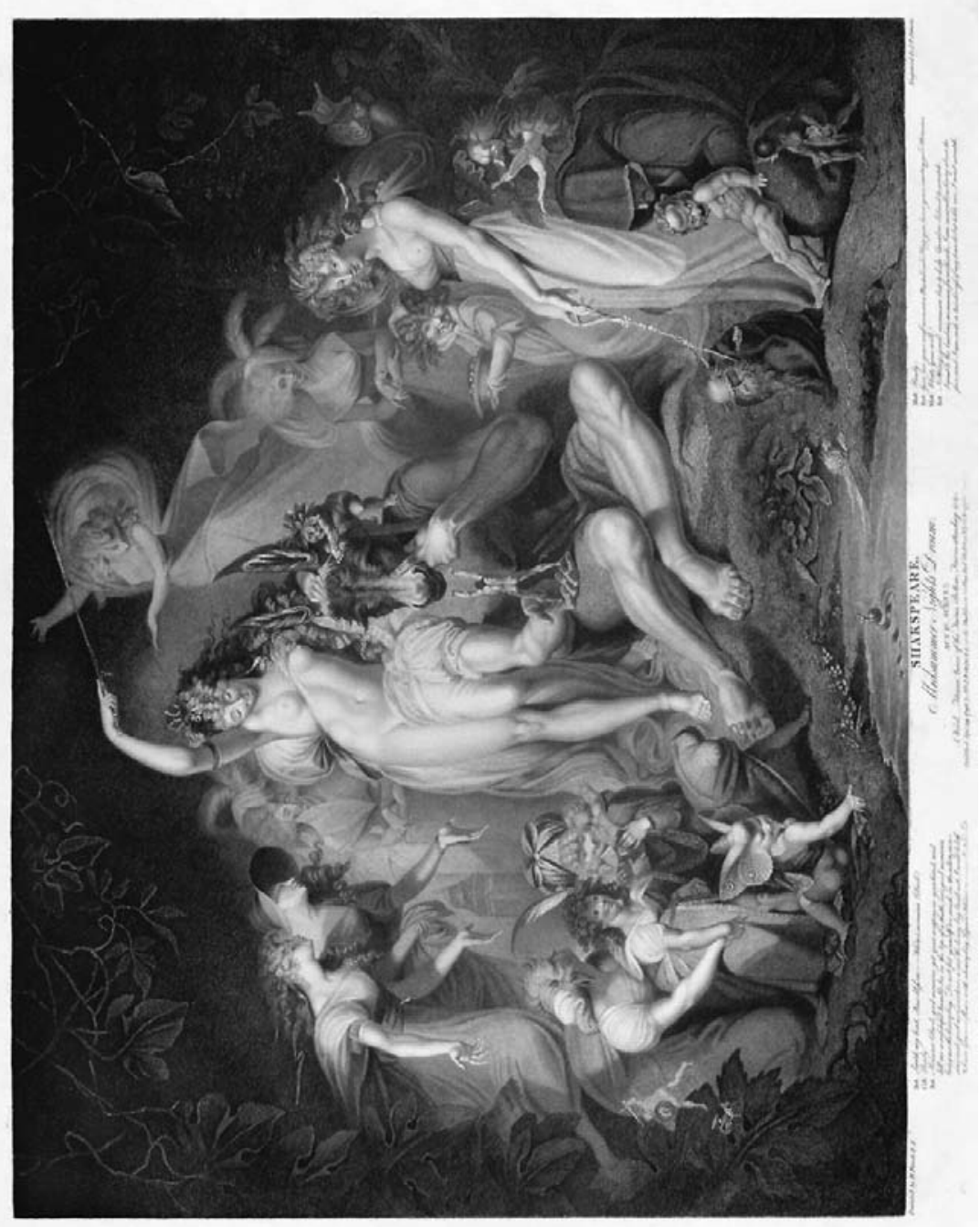

Abb. 9: J. H. Füssli / J. P. Simon, A Midsummer Night's Dream (Act IV, Scene 1): Titania, Queen of the Fairies, Bottom, Fairies Attending 

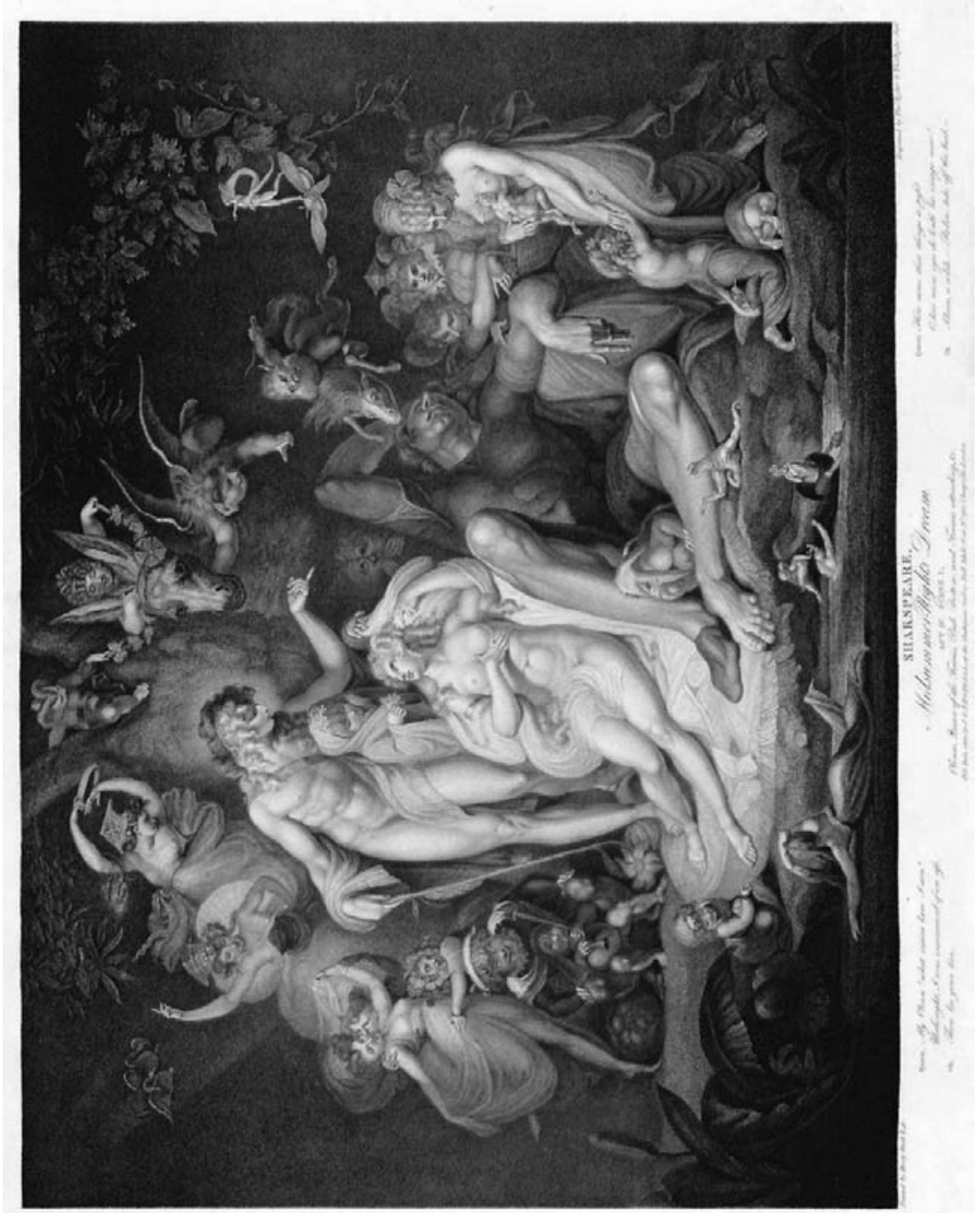

Abb. 10: J. H. Füssli / T. Ryder, A Midsummer Night's Dream (Act IV, Scene 1): Oberon, Queen of the Fairies, Puck. 
Reynolds' Darstellung des Kobolds wirkt ganz harmlos: So einem Wesen kann kein Schaden zugetraut werden; hier ist allenfalls Übermut am Werk, nicht aber Boshaftigkeit. Ganz anders sieht es bei Johann Heinrich Füsslis Bild des Puck aus (Abb. 7). Er zeigt den Puck Robin Goodfellow zwar auch in Gestalt eines Kindes, wie die körperlichen Proportionen vermuten lassen, aber in seinem Gesicht ist deutlich Schadenfreude zu erkennen: Er fliegt durch die Luft und wird in einem Moment gezeigt, in dem er offenbar gerade einen seiner Scherze treibt. Mit seiner rechten Hand deutet der Puck nach oben; seine Geste wird durch einen Kreis verlängert, der zunächst Kugeln zeigt, dann aber - unmittelbar über seinem linken Flügel - einen Halbmond, darunter einen Stern enthält und damit offenbar astrologische Fähigkeiten andeuten soll; mit seiner linken Hand macht der Kobold eine magische Geste in Richtung auf ein Pferd in der rechten Bildecke, das offenbar mit Wassermassen kämpft und versucht, den Reiter abzuschütteln, der sich angstvoll an seinen Hals klammert. In der linken unteren Ecke scheint eine weibliche Figur begleitet von zwei weiteren Figuren in Miniatur mit grotesken Gesichtern nicht einmal zu bemerken, was sich über ihr abspielt. Im Hintergrund des Bildes sind undeutlich Felsvorsprünge wie in einem Gebirge auszumachen: Die Umgebung ist zumindest realistisch skizziert, aber der Puck wird deutlich als ein übernatürliches Wesen gezeigt, das nicht nur aus Übermut handelt, sondern das boshaft ist und wirklichen Schaden anrichten kann. Füsslis Darstellung ist damit deutlich dunkler und grotesker als Reynolds' (Friedman 123); beide zusammengenommen ergänzen einander jedoch, wie dies im Drama mit unterschiedlichen Textstellen der Fall ist.

Füssli trägt jedoch noch zwei weitere Bilder aus dem Themenkreis des Sommernacbtstraums zur Boydell Gallery bei (zu seinen Quellen vgl. Friedman 207-208). Die beiden großformatigen Ölgemälde zeigen Titania vor und nach der Verzauberung Zettels: Das in der Handlungssequenz frühere der beiden Bilder zeigt Titania, bis auf einen herabrutschenden Umhang nackt, in einer wie tänzerischen Pose in der Bildmitte neben Zettel; ihre linke Hand krault ihn zwischen den Ohren, während sie mit der Rechten eine Art Gerte über ihrem Kopf schwingt (Abb. 8). Zettel, den Blick nach unten gerichtet, zeigt Senfsamen, der auf seiner Hand steht, wo er ihn an der Nase kratzen soll; Bohnblüte, der anders als Senfsamen deutliche Züge eines Gnomen hat, sitzt hinter seinem linken aufgestellten Ohr und kratzt ihn dort. Um diese zentrale Figurengruppe herum sind weitere Wesen zu sehen; einige davon mit menschlichen Zügen und in menschlicher Größe, gekleidet in halbtransparente Gewänder und in theatralisch wirkenden Posen, andere kleinwüchsig und mit teils groteskem Aussehen (Maisak 70-72). So wendet sich unten links am Bildrand ein zwergenhafter Elf halb zum Teich im Vordergrund um. An beiden Seiten seines Kopfes wachsen ihm Schmetterlingsflügel: es handelt sich offenkundig um Motte. 
In der rechten Bildecke kauert eine dunkel gekleidet hexenhafte Figur mit tief in das Gesicht gezogener Kapuze über einem Wechselbalg mit dämonischem Gesicht; weiter oben lugt ein ebenfalls dämonenhaft wirkendes Gesicht aus den Blättern hervor. Der Hintergrund des Bildes ist durch die Figuren fast völlig verstellt; der Wald, in dem die Szene spielt, ist lediglich durch die Blätterumrahmung angedeutet (vgl. auch Maisak 70). Die fröhliche Selbstvergessenheit, die sich in Titania zeigt, steht in einem starken Spannungsverhältnis zu den um sie herum verteilten grotesken Wesen. Sogar der indische Knabe, um dessenwillen Oberon und Titania sich streiten, wirkt hier fremdartig; er ist oberhalb von Motte abgebildet, erkennbar an seinem orientalisch wirkenden Turban.

Das Folgebild zeigt Titania unmittelbar nach ihrem Erwachen aus der Verzauberung (Abb. 8). Sie liegt neben Zettel, dessen Eselskopf inzwischen entfernt ist er wird gerade fortgeflogen und ist noch über ihm zu sehen - ; während Zettel zur rechten Bildseite gedreht liegt, ist Titania nach links Oberon zugewandt. Oberon sieht seltsam androgyn aus. Wie Titania in dem chronologisch früheren Bild ist er lediglich mit einem Umhang bekleidet, der sich nur noch über seinem linken Oberschenkel befindet; er neigt sich in einer belehrenden Haltung halb über Titania. Zwischen ihnen steht der indische Knabe. Diese Gruppe wird durch HellDunkel-Kontraste hervorgehoben; Zettel hingegen verschwindet fast im Halbdunkel auf der rechten Bildseite. Um die zentrale Gruppe herum sind Elfen und andere Wesen angeordnet, deren Gestik auf der Seite des Elfenkönigspaares Freude und Ausgelassenheit ausdrückt: Am linken Bildrand küssen sich zwei Elfen, über ihnen haben zwei weitere ihre Arme in tänzerischer Pose erhoben. Auf Zettels Bildseite hingegen dominieren sexuelle Annäherungen (Maisak 71): Am rechten Bildrand drückt eine weibliche Gestalt ein mit Widderhörnern versehenes Wesen zwischen ihre Brüste, halb zwischen ihren Schenkeln kniet ein weiterer Elf.

In beiden Bilder Füsslis sind also gleichzeitig unterschiedliche Aspekte der Elfenwelt dargestellt. Einerseits zeigen die Elfen Freude und unbekümmerte Ausgelassenheit, wie sie auch im Text beschrieben wird, andererseits finden sich klare sexuelle Darstellungen, wie sie im Text allenfalls angedeutet werden. Darüber hinaus sind die Elfen bei weitem nicht nur als schön und kindlich gezeichnet, wie sie Reynolds in seinem Puck-Gemälde zeigt. Sie sind teilweise monströs dargestellt, wie sich dies auch in Füsslis Puck-Darstellung zeigt.

Im Vergleich der Bilddarstellungen aus der Boydell Gallery mit der Textrezeption und Inszenierungspraxis sind also interessante Unterschiede zu erkennen. Das ganze 18. Jahrhundert hindurch wird der Sommernachtstraum nicht in seiner ursprünglichen Gestalt inszeniert, sondern stets in abgewandelten Fassungen, und bis zur Mitte des Jahrhunderts spielen die Elfen dabei keine Rolle. Zentral ist stets die Handwerker-Handlung. Diese wiederum spielt in den Boydell-Illustrationen keinerlei Rolle. Hier hat sich also eine Verschiebung in der Interpretation des Dramas ergeben. Ausweislich der vier in Kupferstichen reproduzierten Gemälde 
sind Elfen jetzt wieder ,hoffähig geworden und für die ShakespeareInterpretatoren interessant.

Ein wesentlicher Grund dafür ist sicherlich in den ,gotischen' Romanen zu sehen, die seit 1765 - dem Erscheinungsjahr von Horace Walpoles Castle of Otranto (Die Burg von Otranto) - das englische Lesepublikum fesseln. Zwar spielt Walpoles Roman nicht in der Elfenwelt, aber er ist voll von unerklärlichen, übernatürlichen Elementen, die eine neue Gattung des Romans begründen. In Walpoles literarischer Nachfolge entstehen Romane wie Ann Radcliffes Mysteries of Udolpho (Geheimnisse von Udolpho) und Matthew Gregory Lewis' The Monk (Der Mönch): Letzterer ist stark beeinflußt von, gotischen' Erzählungen aus Deutschland, die in ihren Handlungen ungleich dunkler sind als vergleichbare englische Romane. Eine ähnliche Diskrepanz in der Interpretation von magisch handelnden Wesen zeigt sich auch in der Gegenüberstellung der beiden Puck-Darstellungen von Reynolds und Füssli (vgl. zu dessen Kunsttheorie Maisak 59-60 sowie Ditchburn-Bosch).

Es ist jedoch nicht die ,schwarze Romantik' deutscher Prägung, die sich als stilbildend und einflußreich für die Inszenierungen des 19. Jahrhunderts herausstellt, sondern eher Reynolds' heitere, spitzbübische Puck-Auffassung: Bis in das letzte Drittel des 19. Jahrhunderts wird der Puck in englischen Inszenierungen durch ein Mädchen dargestellt, das - wie Reynolds' Puck - auf einem Pilz sitzend eingeführt wird; besonders grazil und beeindruckend war in dieser Rolle Ellen Terry, die unter Keans Regie in den 1860er Jahren spielte. Ein letzter Blick auf die Darstellung der Elfen unter Kean zeigt eine seltsame Mischung aus der Vorstellung, die Elfen seien ein halb kriegerisches Volk, wie es sich in der Illustration zu Rowes Shakespeare-Ausgabe von 1709 auf der Seite Oberons zeigt, und aus der verspielten Darstellung, wie sie sich in den ,normalen' Figuren bei Füssli zeigt. Die dunkle(re) Seite, die die Elfen mit dem Kobold Robin Goodfellow durchaus teilen, wird erst in den Bühnendarstellungen des 20. Jahrhunderts wieder greifbar.

Die Illustrationen zum Sommernachtstraum, die für die Boydell Gallery entstanden sind, zeigen also einen Wendepunkt in der visuellen Rezeption des Dramas: Die nicht-menschlichen Wesen, die Elfen und der Kobold, werden zum zentralen Gegenstand erhoben (Mariotti 71), und die Bilder zeigen sie entweder als unschuldige Lichtgestalten oder als fremdartige Kreaturen, deren Körper teils Tier- oder Pflanzengestalt haben. Die Dramenherkunft der Figuren spielt keine Rolle mehr statt dessen haben die Bilddarstellungen zunehmend Einfluß auf die Theaterinszenierungen. Allerdings gilt das nur in geringem Maß für Füsslis düstre Visualisierung des Puck: Diese Interpretation wird erst im zwanzigsten Jahrhundert von den Theaterregisseuren aufgenommen. 


\section{Verwendete Literatur}

Primärliteratur

Garrick, David [and George Colman]. A Fairy Tale. In Two Acts. Taken from Shakespeare [London 1763]. Reprinted in: The Plays of David Garrick. Ed. Gerald M. Berkowitz. 4 vols., hier vol. 3. New York/London: Garland, 1981.

Garrick, David, The Fairies. An Opera. Taken from A Midsummer Night's Dream, Written by Shakespear [London 1755]. Reprinted in: The Plays of David Garrick. Ed. Gerald M. Berkowitz. 4 vols., hier vol. 2. New York/London: Garland, 1981.

Garrick, David. A Midsummer Night's Dream. Written by Shakespeare: With Alterations and Additions, and Several New Songs [London 1763]. Reprinted in: The Plays of David Garrick. Ed. Gerald M. Berkowitz. 4 vols., hier vol. 3. New York/London: Garland, 1981.

Leveridge, Richard. The Comick Masque of Pyramus and Thishe. As it is Perform'd at the Theatre in Lincoln's-Inn Fields. London: Mears, 1716.

[Leveridge, Richard]. Pyramus and Thishe: A Mock-Opera. Written by Shakespeare. Set to Musick by Mr. Lampe. London: Woodfall, 1745.

[Settle, Elkanah?]. The Fairy-Queen: An Opera. Represented at the Queen's Theatre by their Majesties Servants. With Alterations, Additions, and Several New Songs, London: Tonson, 1693.

Shakespeare, William, A Midsummer-Night's Dream. A Comedy. The Works of Mr. William Shakespear. Ed. Nicholas Rowe and Charles Gildon. 7 vols, hier vol. 2 (1709). London: Tonson, 1709-1710.

Shakespeare, William. The Works of Shakespeare. Ed. Thomas Hanmer. Oxford: Clarendon Press, 1770. 6 vols.

Shakespeare, William. Bell's Edition of Shakespeare's Plays. London: Bell, 1773-1774. 9 vols.

Sekundärliteratur

Allen, Brian. „The Early Illustrators of Shakespeare“. Shakespeare in Art. Ed. J. Martineau and Desmond Shawe-Taylor. London: Merrell, 2003. 49-59.

Bruntjen, Sven H. A. John Boydell, 1719-1804: A Study of Art Patronage and Publishing in Georgian London. New York/London: Garland, 1985.

Campbell, Margaret. Henry Purcell: Glory of His Age, Oxford: Oxford University Press, 1995.

Ditchburn-Bosch, Ursula. Johann Heinrich Füsslis Kunstlehre und ibre Auswirkung auf die Shakespeare-Interpretation, Zürich: Juris, 1960. 
Friedman, Winifred H. Boydell's Shakespeare Gallery. New York/London: Garland, 1976.

Gerkens, Dorothea. Elfenbilder - Traum, Rausch und das Unbewusste. Die Erkundung des menschlichen Geistes in der Malerei des 18. und 19. Jahrhunderts veröffentlicht. Berlin: Reimer, 2009.

Gildon, Charles (1710a). „Essay on the Art, Rise and Progress of the Stage in Greece, Rome and England“. The Works of Mr. William Shakespeare. 7 vols., hier vol. 7 (1710). Ed. Nicholas Rowe. London: Tonson, 1709-1710. i-lxvii.

Gildon, Charles (1710b). „Remarks on the Plays of Shakespear“. The Works of Mr. William Shakespeare. 7 vols., hier vol. 7 (1710). Ed. Nicholas Rowe. London: Tonson, 1709-1710. 257-444.

Graham, Arthur. Shakespeare in Opera, Ballet, Orchestral Music, and Song. An Introduction to Music Inspired by the Bard. Lewiston/Queenston/Lampeter: Edward Mellen Press, 1997.

Hammerschmidt-Hummel, Hildegard. „Boydell's Shakespeare Gallery and its Role in Promoting English History Painting“. The Boydell Shakespeare Gallery. Ed. W. Pape / F. Burwick. Bottrop: Pomp, 1996. 33-44.

Hammerschmidt-Hummel, Hildegard. Die Shakespeare-Illustration (1594-2000). Bildkünstlerische Darstellungen zu den Dramen William Shakespeares: Katalog, Geschichte, Funktion und Deutung. 3 Teile. Wiesbaden: Harrassowitz, 2003.

Kennedy, Judith M.. „Oberon Viewed in the Nineteenth Century“. Shakespeare and the Visual Arts. Hg. H. Klein, J. L. Harner. Lewiston/Queenston/Lampeter: Edwin Mellon Press, 2000. 336-353.

Lürig, Heiner, und Heinz Rudolf Kunze. Ein Sommernachtstraum. Das Musical nach William Shakespeare. Göttingen: Satzwerk, 2004.

Maisak, Petra. „Henry Fuseli - 'Shakespeare's Painter". The Boydell Shakespeare Gallery. Ed. W. Pape / F. Burwick. Bottrop: Pomp, 1996. 57-74.

Mariotti, Giovanni. „Dreams on Stage: Füssli and Shakespeare“. FMR 89 (1997). 60-84.

Muller, Frans, and Julie Muller. „Completing the Picture: the Importance of Reconstructing Early Opera“. Early Music 33 (2005). 667-682.

Pepys, Samuel. The Diary of Samuel Pepys. Ed. Robert Latham and William Matthews. 11 vols, hier vol. 3: 1662 (1970). London: Bell, 1970-1983.

Pfister, Manfred. „Die heiteren Komödien“. Shakespeare-Handbuch: Die Zeit, der Mensch, das Werk, die Nachwelt. Hg. Ina Schabert. Stuttgart: Kröner, 2000. 381439.

Postle, Martin. Sir Joshua Reynolds: The Subject Pictures. Cambridge: Cambridge University Press, 1995.

[Proposal Mr. Alderman Boydell, Josiah Boydell, and George Nicol, propose to publish by subscription a most magnificent and accurate edition [...7. London: s.l., 1786. 
Shawe-Taylor, Desmond. „Theatrical Painting from Hogarth to Fuseli“. Shakespeare in Art. Ed. J. Martineau and Desmond Shawe-Taylor. London: Merrell, 2003. 115-173.

Stone, George Winchester. „A Midsummer Night's Dream in the Hands of Garrick and Colman“. Publications of the Modern Language Association of America 54 (1939): 467-482. 